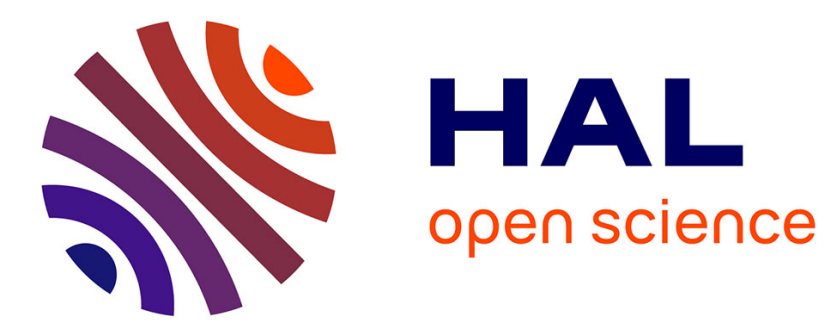

\title{
Application de la prospection magnétotellurique artificielle à l'étude de la profondeur d'un sol
}

Philippe Mérot, Chantal Gascuel, Georges Chevassu

\section{To cite this version:}

Philippe Mérot, Chantal Gascuel, Georges Chevassu. Application de la prospection magnétotellurique artificielle à l'étude de la profondeur d'un sol. Agronomie, 1986, 6 (1), pp.57-66. hal-00884848

\section{HAL Id: hal-00884848 \\ https://hal.science/hal-00884848}

Submitted on 1 Jan 1986

HAL is a multi-disciplinary open access archive for the deposit and dissemination of scientific research documents, whether they are published or not. The documents may come from teaching and research institutions in France or abroad, or from public or private research centers.
L'archive ouverte pluridisciplinaire HAL, est destinée au dépôt et à la diffusion de documents scientifiques de niveau recherche, publiés ou non, émanant des établissements d'enseignement et de recherche français ou étrangers, des laboratoires publics ou privés. 


\title{
Application de la prospection magnéto- tellurique artificielle à l'étude de la profondeur d'un sol
}

\author{
Philippe MEROT, Chantal GASCUEL-ODOUX \& Georges CHEVASSU (*) \\ I.N.R.A., Laboratoire de Recherches de la Chaire de Science du Sol, E.N.S.A., 65 route de Saint-Brieuc, \\ F 35042 Rennes Cedex \\ $\left(^{*}\right)$ Laboratoire Régional de l’Équipement, 12 rue Sully, F 22000 Saint-Brieuc
}

\begin{abstract}
Une méthode de prospection géophysique, la magnéto-tellurique artificielle (M.T.A.), a été appliquée à la prospection des sols à grande échelle. Dans un premier temps, un traitement géostatistique des données issues de la M.T.A. et de l'étude de la profondeur du sol a permis de mettre en évidence une bonne corrélation entre ces variables. Dans un deuxième temps, la carte des sols a pu être précisée, en s'appuyant sur les enregistrements de résistivité apparente M.T.A.

En conclusion, on préconise l'emploi de cette méthode de prospection géophysique comme méthode préalable à la cartographie des sols dans les cas où une connaissance précise du sol et du sous-sol est nécessaire (parcelles expérimentales, essais culturaux, étude de variabilité spatiale...).
\end{abstract}

Mots clés additionnels : Prospection géophysique, cartographie des sols, variabilité spatiale.

A geophysical method, artificial magneto-tellurics (M.T.A.), has been applied to large scale soil surveys. First, a geostatistical treatment of data from M.T.A. and from studies on soil depth showed good fit between these datasets. Second, soil maps could be clarified by taking M.T.A. data into account. As a conclusion, we recommend the use of this geophysical method as a tool prior to large-scale mapping.

Additional key words : Geophysical prospecting, soil mapping, spatial variability.

\section{INTRODUCTION}

Les méthodes géophysiques permettent d'obtenir des informations globales sur le sol et le sous-sol. Parmi ces méthodes, la Magnéto-Tellurique-Artificielle (M.T.A.) permet de mesurer la résistivité apparente des terrains de sub-surface : pour cela, on mesure simultanément le champ magnétique et le champ électrique, sur une fréquence donnée d'une onde électro-magnétique. Les variations de résistivité apparente que l'on va interpréter dépendent avant tout des contrastes de résistivité que l'on rencontre entre un matériau conducteur (par exemple un sol ou une arène) et un matériau non conducteur (un granite ou un schiste sain) ainsi que des variations d'épaisseur du niveau supérieur. Elle dépendra également de la quantité d'eau et de la composition chimique de l'eau contenue dans le sol (annexe 1).

En prospection M.T.A., on déplace le capteur le long d'un axe de façon à suivre les variations latérales de résistivité apparente. La profondeur de pénétration de l'onde est fonction de la fréquence choisie et de la résistivité du milieu. De ce fait, l'épaisseur prospectée varie dans le même sens que la résistivité, ce qui a pour effet de contraster les résultats. En imposant une fréquence, on choisit une gamme de profondeur de pénétration: pour une résistivité apparente de $100 \Omega \mathrm{m}$, la pénétration sera de $40 \mathrm{~m}$ pour une fréquence de $15 \mathrm{kHz}$, de $13 \mathrm{~m}$ pour $164 \mathrm{kHz}$, de $6 \mathrm{~m}$ pour $700 \mathrm{kHz}$. La profondeur d'investigation, définie comme la profondeur effective à laquelle une anomalie peut être détectée, se situe entre 0,3 et 0,5 fois la profondeur de pénétration (LAGABRIELLE et al., 1983).

Cette méthode développée au C.N.R.S. (inventeurs GUINEAU \& DUPIS, 1973) a été mise en œuvre ces dernières années par les Laboratoires des Ponts et Chaus- 
sées pour ses applications géotechniques, lors de tracés routiers, de recherche de matériau et d'étude de projets des tranchées (LAGABRIELLE et al., 1983).

En Science du Sol, diverses applications ont été tentées. Ainsi AurousSEAU (1976) a réussi avec la M.T.A. à repérer la profondeur du toit du granite sain, sous un manteau d'altération, sur une séquence de sol du Morvan. Cependant, les fréquences d'ondes alors utilisables $(15 \mathrm{kHz})$ ne permettaient que des investigations sur de fortes épaisseurs de terrain relativement à l'épaisseur d'un sol et non des applications à des zones plus superficielles. Des reconnaissances faites à Rennes (MEROT, 1980, non publiées), à l'aide d'émetteur de fréquences $15 \mathrm{kHz}$ et $164 \mathrm{kHz}$ sur des sols bruns sur schistes briovériens donnent des résultats ayant trait plus aux structures géologiques qu'au sol lui-même (fig. 1).

La mise au point récente de capteurs capables de recevoir les ondes moyennes (fréquence de l'ordre de $1 \mathrm{MHz}$ ) permet d'envisager l'application de la M.T.A. à l'étude des premiers mètres du sol. Ainsi, BOTTRAUD (1983) a appliqué la M.T.A. avec différentes fréquences ( 1242 et $218 \mathrm{kHz}$ ) à la prospection cartographique de différents sols : sols fersiallitiques avec encroûtements calcaires; sols fersiallitiques lessivés sur cailloutis ; sols sur alluvions et altérites. Il montre les différentes possibilités et limites de cette méthode dans les cas étudiés.

L'objectif du travail présenté ici est de tester les possibilités de reconnaissance de la couverture pédologique à grande échelle par la M.T.A. à fréquence élevée, en confrontant l'information issue de cette méthode aux données issues de la cartographie des sols. La M.T.A. faisant apparaître de grands contrastes de résistivité là où existent des discontinuités horizontales franches, nous avons choisi de comparer les données M.T.A. à la variation de la profondeur du contact entre, d'un côté, les horizons superficiels et l'altérite sans éléments grossiers et, de l'autre, l'altérite grossière et le schiste.

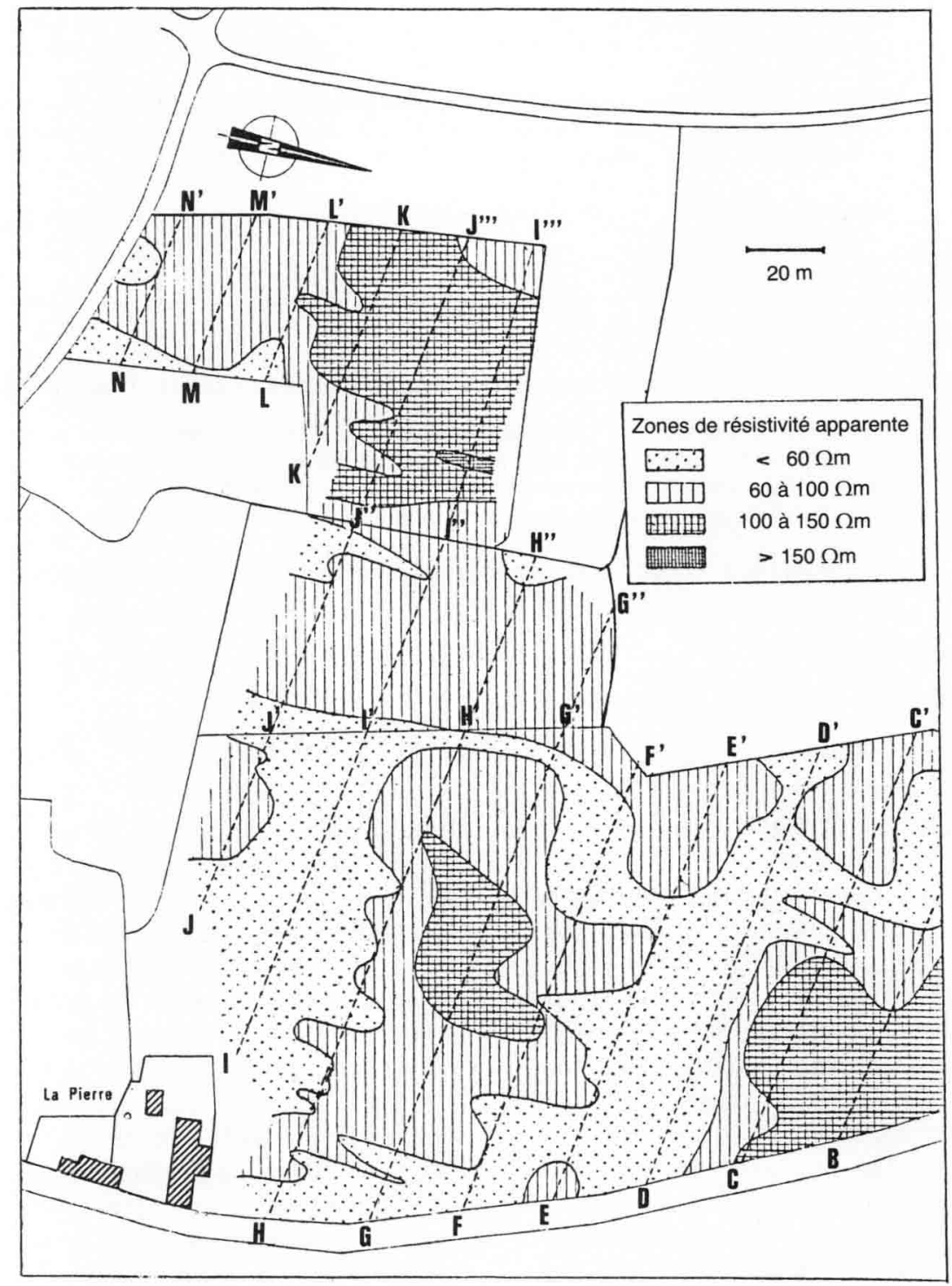




\section{MATÉRIEL ET MÉTHODE}

\section{A. Le milieu pédologique}

Le travail a été mené sur 4 ha du bassin versant élémentaire de Nouvoitou, dans la région de Rennes (fig. 2). Les sols sont développés dans des limons acides sur schistes briovériens. A l'amont, les sols sont sains, bruns faiblement lessivés, tandis qu'à l'aval ils sont hydromorphes, bruns lessivés et dégradés. Le schiste se présente à l'amont sous forme de plaquettes revêtues d'argile, ménageant une porosité grossière entre les graviers. Vers l'aval, on passe à une altérite de schiste limono-argileuse, généralement riche en graviers, à structure massive et à porosité faible (GASCUEL-ODOUX et al., 1982). De plus, par endroits, on rencontre une altérite sans gravier, au toucher talqueux, nommée par la suite altérite franche.

Les caractéristiques physico-chimiques d'un profil de sol d'amont et d'aval sont rapportées tableau 1. On constate le long des profils l'enrichissement en argile, en sable grossier et l'augmentation de la densité apparente en profondeur.

\section{B. Les conditions d'humidité de la campagne M.T.A.}

Lors de la campagne de mesure M.T.A. (24 et 25 mai 1982), les sols du bassin étaient, en amont, à la capacité au champ ; en aval, ils étaient saturés en surface. La figure 2 présente l'étendue des zones saturées.

\section{Mesures et méthodes de traitement de données}

L'appareil de mesure M.T.A. utilisé est composé d'une antenne (capteur magnétique), d'un tapis de caoutchouc où sont noyées les électrodes, d'un boîtier électronique avec enregistrement graphique. Le tout est tracté par un véhicule tout-terrain léger à la vitesse de $3 \mathrm{~km} / \mathrm{h}$. L'émetteur utilisé était Radio Armorique $(711 \mathrm{kHz})$. Plusieurs séries de trainées, parallèles et distantes de $10 \mathrm{~m}$, ont été réalisées sur 4 ha du bassin (fig. 3). La direction du capteur était dans l'axe de la direction de l'émetteur. Ceci a permis d'obtenir une carte des résistivités apparentes au 1/1000.

Dans un premier temps, les enregistrements M.T.A. ont été discrétisés suivant un pas de $10 \mathrm{~m}$, puis cartographiés par différentes méthodes; cartographie manuelle; cartographie automatique à partir des données estimées aux nœuds d'une grille régulière de pas $5 \mathrm{~m}$. La méthode d'estimation utilisée est le krigeage, basée sur la théorie des variables régionalisées (MATHERON, 1965), appliquée récemment en Science du Sol (Burgess \& WeBster, 1980 ; GASCUELODOuX, 1984) (annexe 2). Cette méthode de traitement géostatistique des données a permis, d'une part, d'obtenir des estimations de la résistivité apparente, de la profondeur du sol et de leur écart-type d'estimation sur des mêmes sites et donc d'obtenir des données aisément comparables. Elle a, d'autre part, permis de visualiser la cohérence des hétérogénéités spatiales de ces 2 paramètres, sous forme cartographique.

Dans un deuxième temps, une reconnaissance pédologique a été faite sur les points où des anomalies de résistivité avaient été mesurées, alors qu'aucune variation pédologique n'avait été préalablement reconnue.

TABLEAU 1

Granulométrie, densité apparente et capacité d'échange. Profil de versant et profil d'aval à Nouvoitou (in PePIN \& GAREL, 1980). Granulometry, bulk density and exchange capacity of two soil profiles (up and down slope; Nouvoitou). PROFIL AMONT

\begin{tabular}{|c|c|c|c|c|c|c|c|c|c|c|c|c|c|}
\hline \multirow{2}{*}{ Horizon } & \multirow{2}{*}{$\begin{array}{l}\text { Profondeur } \\
\mathrm{cm}\end{array}$} & \multicolumn{5}{|c|}{ Granulométrie \% $\%$} & \multirow{2}{*}{$\begin{array}{c}\text { Densité } \\
\text { apparente }\end{array}$} & \multicolumn{6}{|c|}{ Capacité d'échange meq pour $100 \mathrm{~g}$} \\
\hline & & A & $\mathrm{LF}$ & LG & $\mathrm{SF}$ & SG & & $\mathrm{Ca}^{++}$ & $\mathrm{Mg}^{++}$ & $\mathrm{K}^{+}$ & $\mathrm{Na}^{+}$ & $\mathrm{T}$ & $\mathrm{S} / \mathrm{T} \%$ \\
\hline Ap 1 & $0-5 \mathrm{~cm}$ & 16,6 & 28,0 & 42,7 & 8,0 & 4,7 & & 5,2 & 0,75 & 0,250 & 0,111 & 9,9 & 64 \\
\hline Ap 1 & $6-10$ & 17,5 & 29,2 & 41,6 & 6,8 & 4,9 & 1,20 & 5,5 & 0,55 & 0,080 & 0,152 & 8,7 & 72 \\
\hline Ap 2 & $10-30$ & 16,9 & 28,2 & 42,5 & 7,1 & 5,3 & 1,46 & 5,7 & 0,59 & 0,080 & 0,111 & 8,7 & 74 \\
\hline A 2 & $30-40$ & 18,0 & 28,3 & 42,9 & 6,3 & 4,5 & 1,51 & 5,2 & 0,59 & 0,070 & 0,082 & 7,4 & 80 \\
\hline A 2 & $40-60$ & 19,2 & 28,8 & 42,2 & 5,7 & 4,1 & 1,28 & 5,3 & 0,52 & 0,070 & 0,062 & 7,9 & 75 \\
\hline $\mathrm{Bt}$ & $60-75$ & 24,7 & 26,8 & 39,9 & 3,9 & 4,7 & 1,54 & 7,3 & 0,74 & 0,134 & 0,062 & 9,2 & 89 \\
\hline $\mathrm{Bt}$ & $75-90$ & 22,8 & 26,8 & 27,4 & 8,0 & 15,0 & 1,54 & 6,9 & 0,97 & 0,128 & 0,058 & 9,4 & 85 \\
\hline Btxg & $90-100$ & 20,7 & 29,9 & 25,3 & 9,9 & 14,2 & 1,75 & 5,5 & 1,32 & 0,094 & 0,090 & 8,4 & 83 \\
\hline \multicolumn{14}{|c|}{ PROFIL AVAL } \\
\hline A 11 & $0-10$ & 17,3 & 30,3 & 41,6 & 8,8 & 2,0 & 1,11 & & & & & & \\
\hline A 11 & $10-20$ & & & & & & 1,23 & 4,1 & 0,44 & 0,091 & 0,058 & 7,6 & 62 \\
\hline A 11 & $20-28$ & 18,9 & 29,8 & 42,7 & 6,5 & 2,1 & 0,81 & & & & & & \\
\hline A 22 & $30-38$ & 16,5 & 29,4 & 43,1 & 6,8 & 4,2 & 1,16 & 3,6 & 0,56 & 0,148 & 0,053 & 6,1 & 71 \\
\hline A 22 & $40-50$ & 25,2 & 27,0 & 41,0 & 5,2 & 1,6 & 1,37 & 3,0 & 0,75 & 0,119 & 0,068 & 5,1 & 77 \\
\hline Btgd & $60-80$ & 25,4 & 35,4 & 22,6 & 8,0 & 8,6 & 1,56 & 4,8 & 3,27 & 0,163 & 0,192 & 8,6 & 98 \\
\hline Btgd & $80-100$ & 24,5 & 37,3 & 13,6 & 12,1 & 12,5 & 1,59 & 5,8 & 4,16 & 0,110 & 0,329 & 8,6 & 121 \\
\hline $\mathrm{B} / \mathrm{C}$ & $100-120$ & 20,2 & 42,9 & 15,8 & 9,4 & 11,7 & 1,66 & 13,1 & 8,0 & 0,096 & 0,673 & 10,5 & 208 \\
\hline $\mathrm{C}_{\mathrm{lj}}$ & $\begin{array}{l}160-170 \\
\text { caillouteux }\end{array}$ & 23,0 & 39,4 & 15,1 & 14,9 & 7,6 & 1,77 & & & & & & \\
\hline
\end{tabular}




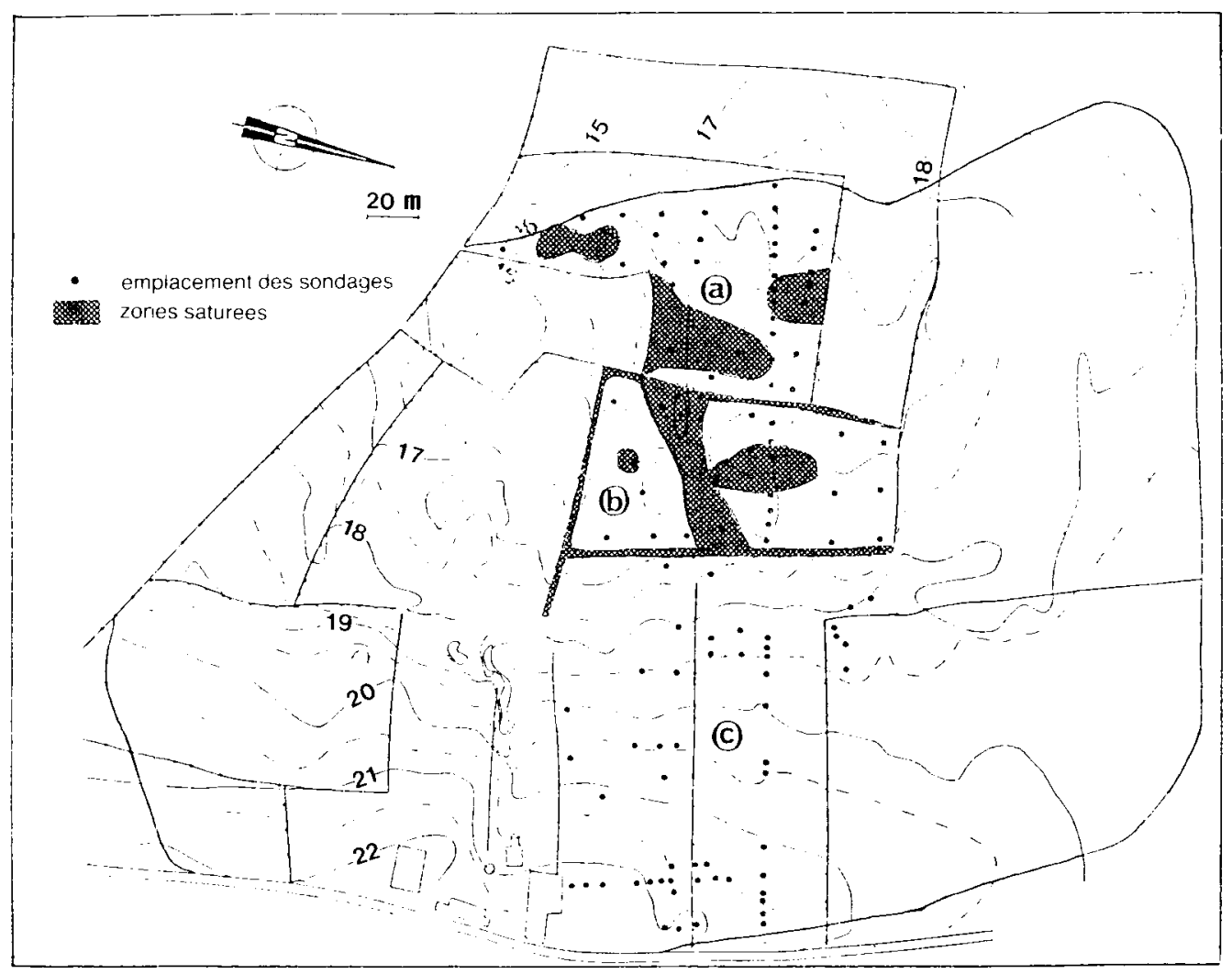

Figure 2

Carte du bassin versant de Nouvoitou : topographie, emplacement des sondages pédologiques sur la partie étudiée $(\bullet)$; zone de saturation apparente des sols lors de la prospection géophysique (en grisé).
Map of the watershed of Nouvoitou : topography, location of soil profiles on the part studied (๑); apparent saturated areas on soil during geophysical prospection (greyish).

Figure 3

Emplacement des traînées de la prospection magnéto-tellurique artificielle (mai 1983 - Nouvoitou).

Location of the artificial magneto-telluric prospection (May 1983 watershed of Nouvoitou).

\section{RÉSULTATS}

\section{A. La carte de profondeur du sol}

La carte de profondeur du sol a été établie à partir de la prospection pédologique menée en 1981 (BRANCHARD \& GASCUEL-ODOUX, 1981) : 113 profils ont été analysés. La profondeur étant une variable régionalisée, on a recherché la structure des mesures obtenues. Au variogramme expérimental, on a ajusté un modèle linéaire (fig. 4), à partir duquel les estimations ont été faites. L'écart-type d'estimation de la profondeur du sol est de l'ordre de $15 \mathrm{~cm}$.

Le champ a, qui correspond au versant orienté à l'est, présente une forte variation de l'épaisseur du sol : le sol s'épaissit dans le sens de la pente, de moins de $40 \mathrm{~cm}$ jusque $80 \mathrm{~cm}$. Le champ b correspond à une zone de bas-fond, plane, avec des sols hydromorphes, 


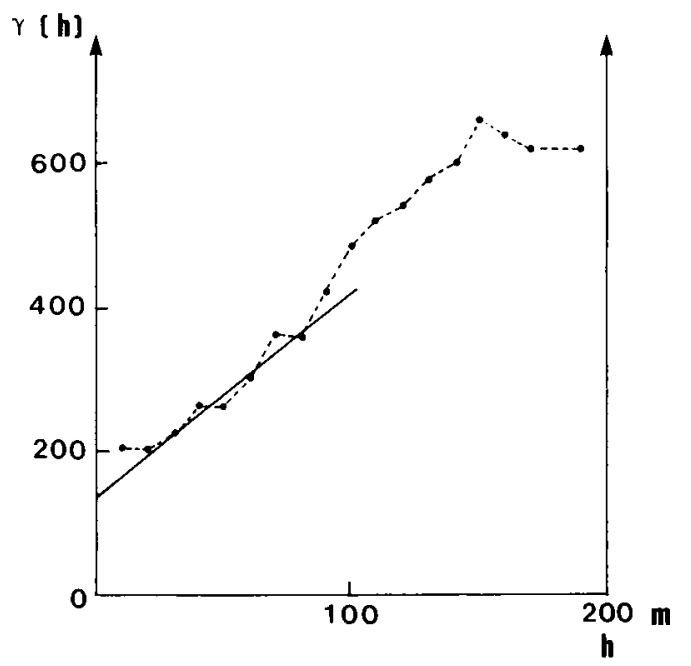

Figure 4

Semi-variogramme de la profondeur du sol.

Semi-variogram of soil depth.

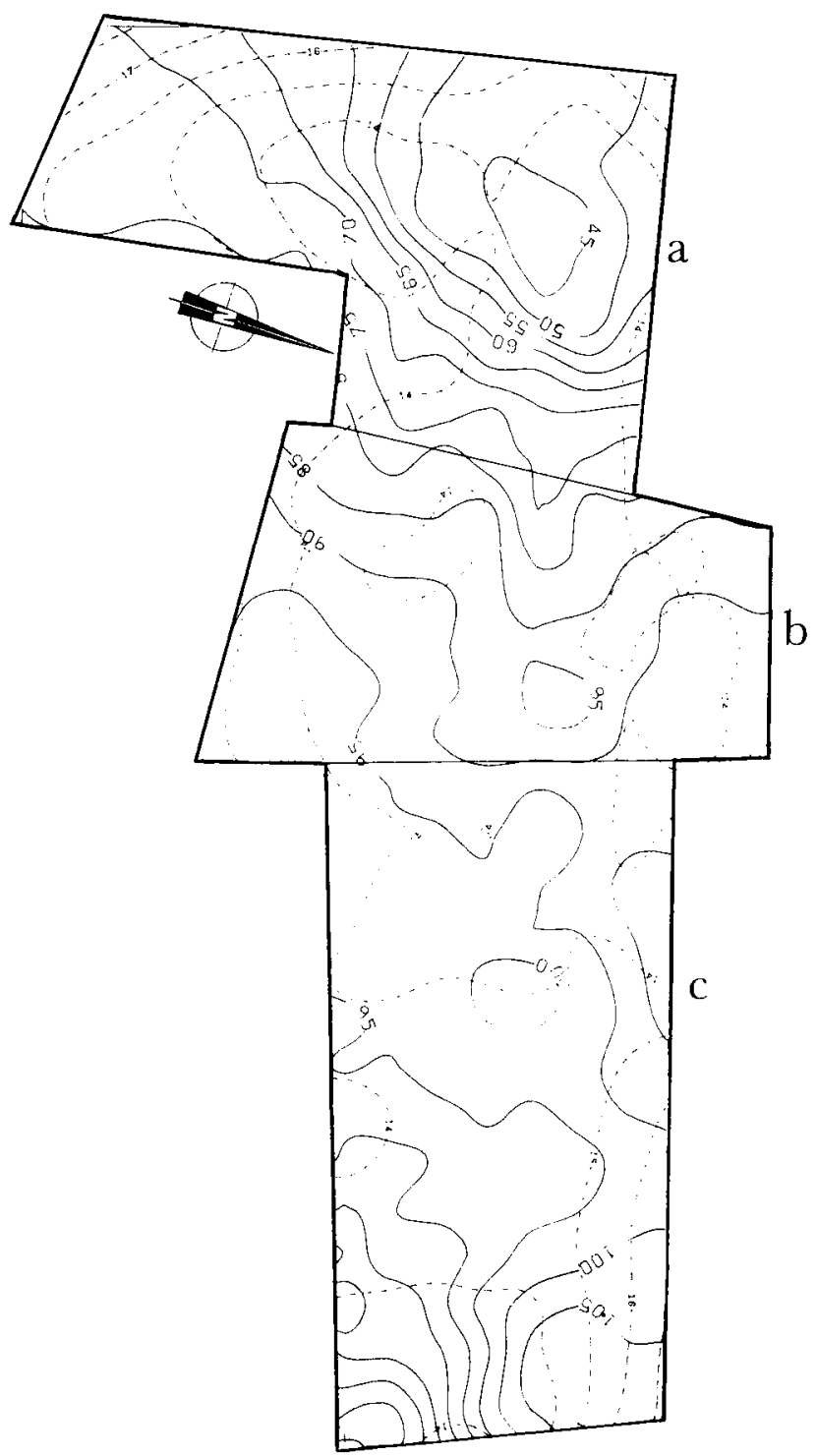

Figure 5

Carte de la profondeur du sol (trait continu) et de l'écart-type associé (trait pointillé).

Map of soil depth (full line) and of its estimated standard deviation (dashed line).

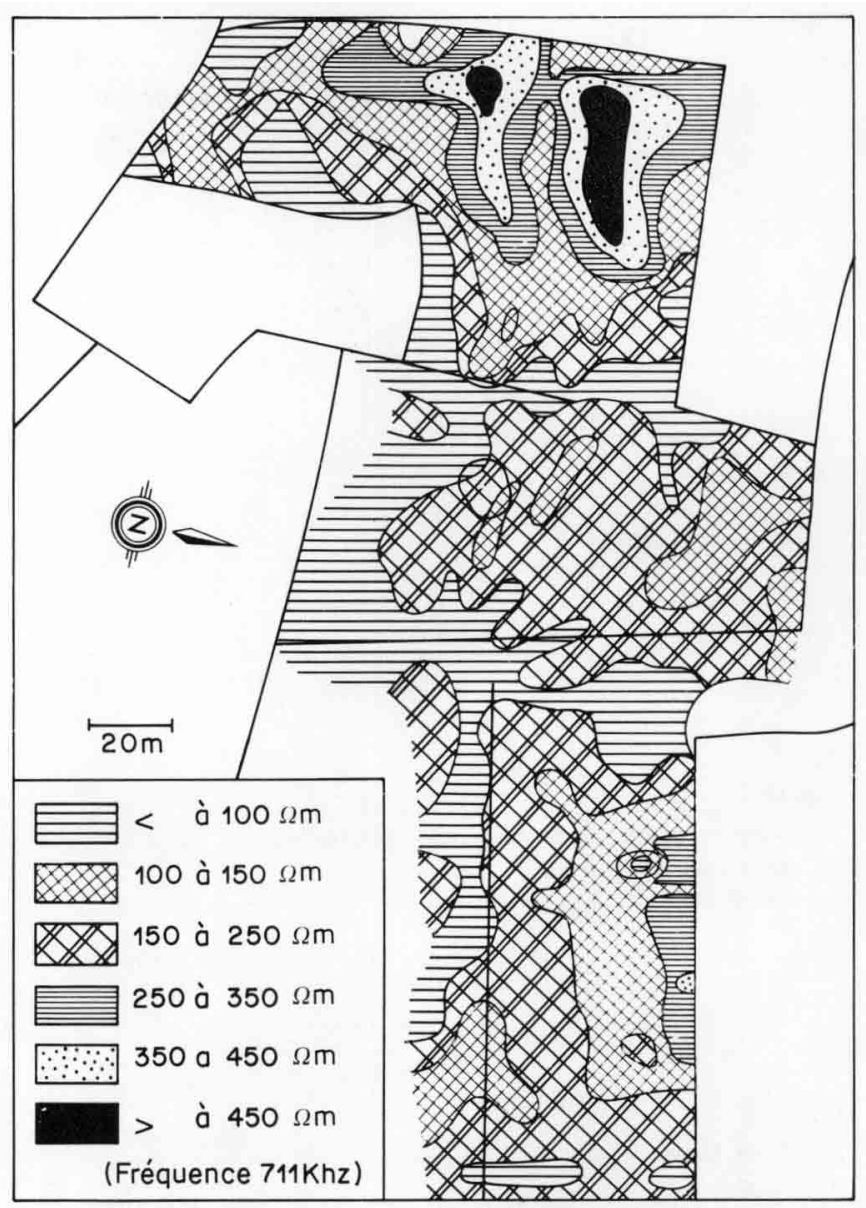

Figure 6

Carte des résistivités apparentes, mesurées à la fréquence de $711 \mathrm{kHz}$ (tracé manuel).

Map of the apparent resistivity, measured at $711 \mathrm{kHz}$ (hand drawn).

dont la profondeur est relativement constante et d'ailleurs assez mal reconnue lors de la prospection. Le champ c correspond au versant orienté à l'ouest. C'est un versant long, aux sols épais de 100 à $120 \mathrm{~cm}$, semblant cependant présenter une remontée du substratum dans son angle ouest (fig. 5).

\section{B. La carte des résistivités apparentes}

Les données M.T.A. ont été discrétisées en 280 points, à partir d'enregistrements continus. Une $1^{\text {re }}$ carte manuelle a été dressée. L'opérateur s'est manifestement fait influencer par le sens des enregistrements faisant apparaitre 2 directions principales pour les limites, directement liées au sens de déplacement du capteur (fig. 6).

Ces données ont été reprises dans un traitement géostatistique. L'histogramme des données expérimentales étant nettement log-normal, nous avons également analysé celles-ci à partir des valeurs logarithmiques. Au variogramme des données expérimentales a été ajusté un modèle linéaire ; de même, un modèle linéaire a été choisi pour les valeurs logarithmiques des données (fig. 7).

Les cartes des estimations à partir des données brutes ou logarithmiques sont peu différentes. Cependant, les tests de robustesse effectués sont meilleurs 


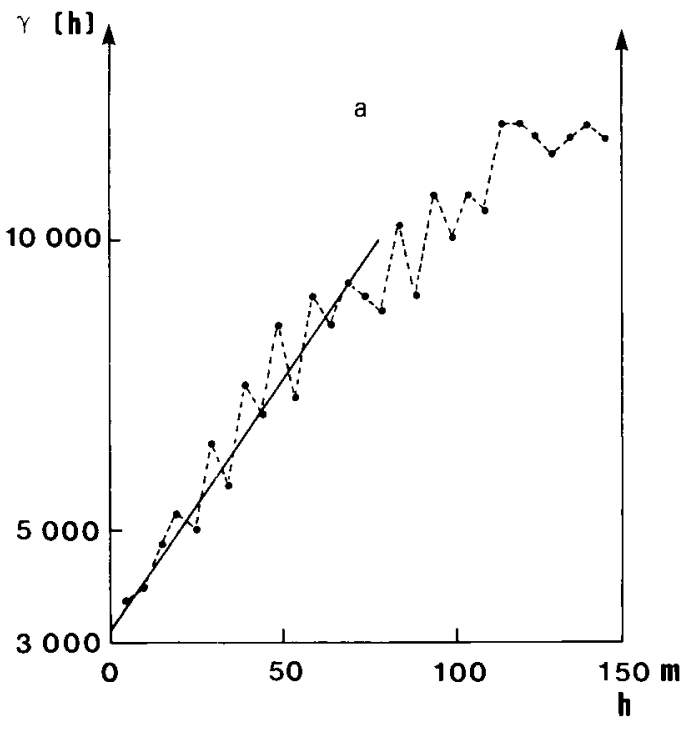

Figure 7

Semi-variogramme de la résistivité apparente:

a) sur les données brutes,

b) sur les données log normales.

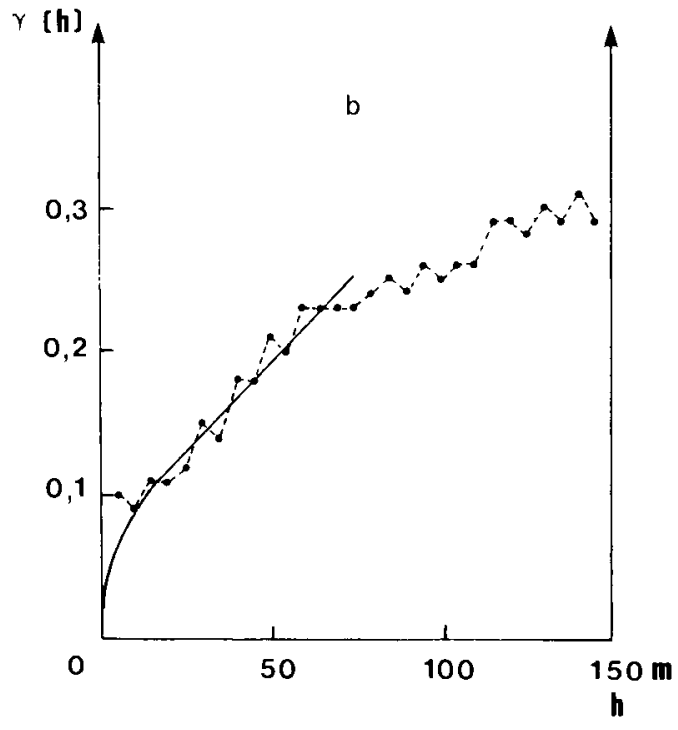

Semi-variogram of apparent resistivity :

a) based on raw data,

b) based on log normal data. sur les données logarithmiques que sur les données brutes et indiquent donc une meilleure reconnaissance des données ponctuelles dans ce cas, ce qui est normal (fig. $8 a$ et $8 b$ ) (annexe 1).

Les cartes de l'estimation de la résistivité apparente présentent dans le champ a un fort gradient avec une zone à très forte résistivité $(450 \Omega \mathrm{m})$; le champ b a une relative homogénéité de valeur; le champ c présente une structure plus complexe : zone à forte résistivité sur sa limite nord entourée par une zone à résistivité décroissante, jusqu'à $100 \Omega \mathrm{m}$, à l'aval. On remarque également une anomalie très localisée de faible résistivité dans une zone à forte résistivité. L'écarttype est constant et égal à $60 \Omega \mathrm{m}$.

\section{COMPARAISON M.T.A. - PROFONDEUR AU SOL}

- Une $1^{\text {re }}$ comparaison a été faite entre la carte de la profondeur estimée et la carte de la résistivité apparente estimée. On constate une concordance variable entre les 2 cartes selon la zone observée. Le champ a présente une concordance remarquable entre zone à forte résistivité et zone à faible épaisseur. Le coefficient de corrélation a été calculé entre les logarithmes des valeurs de la profondeur et ceux de la résistivité apparente sur 120 estimations aux nœuds de la grille (C $=-0,91$ ) (fig. 9).

Sur le champ b et surtout sur le champ c, les concordances entre les 2 cartes sont beaucoup moins bon- nes. Pour la partie sud du champ c, le gradient de profondeur et le gradient de résistivité semblent liés : augmentation de la profondeur et diminution de la résistivité apparente le long de la pente. Par contre, la partie nord du champ c présente à la fois des variations importantes et des anomalies locales de résistivité qui ne correspondent à rien sur la carte des profondeurs. Cependant, la densité d'information M.T.A. étant beaucoup plus forte que celle acquise sur la profondeur du sol et au vu des résultats sur le champ a, il semble qu'il faille chercher une réponse au niveau de la précision de la carte du sol. Par ailleurs, on n'a pas mis en évidence de modification de la résistivité apparente liée à l'humidité des sols. Ce dernier point peut s'expliquer par les faibles écarts d'humidité des sols entre l'état ressuyé et l'état saturé. Ces variations induisent des différences de résistivité apparente beaucoup plus faibles que celles dues au matériau constitutif du sol.

- Une $2^{\mathrm{e}}$ comparaison a été faite sur sondage postérieur à la prospection M.T.A. : des sondages pédologiques ont été réalisés selon des axes choisis parce qu'ils recoupaient les variations de résistivité apparente alors que la carte des sols, préalablement établie, ne laissait apparaître aucune variation de la profondeur du sol (fig. 10). On constate effectivement une bonne concordance entre les variations du toit de schiste et les variations de résistivité apparente.

- Une dernière étape a consisté à réintroduire les sondages pédologiques faits en fonction de la M.T.A., ce qui a permis d'améliorer la concordance entre les 2 cartes de la profondeur du sol et de la résistivité M.T.A. dans le champ c, au niveau de la zone de forte résistivité et de la petite anomalie faiblement résistive (fig. 11). 


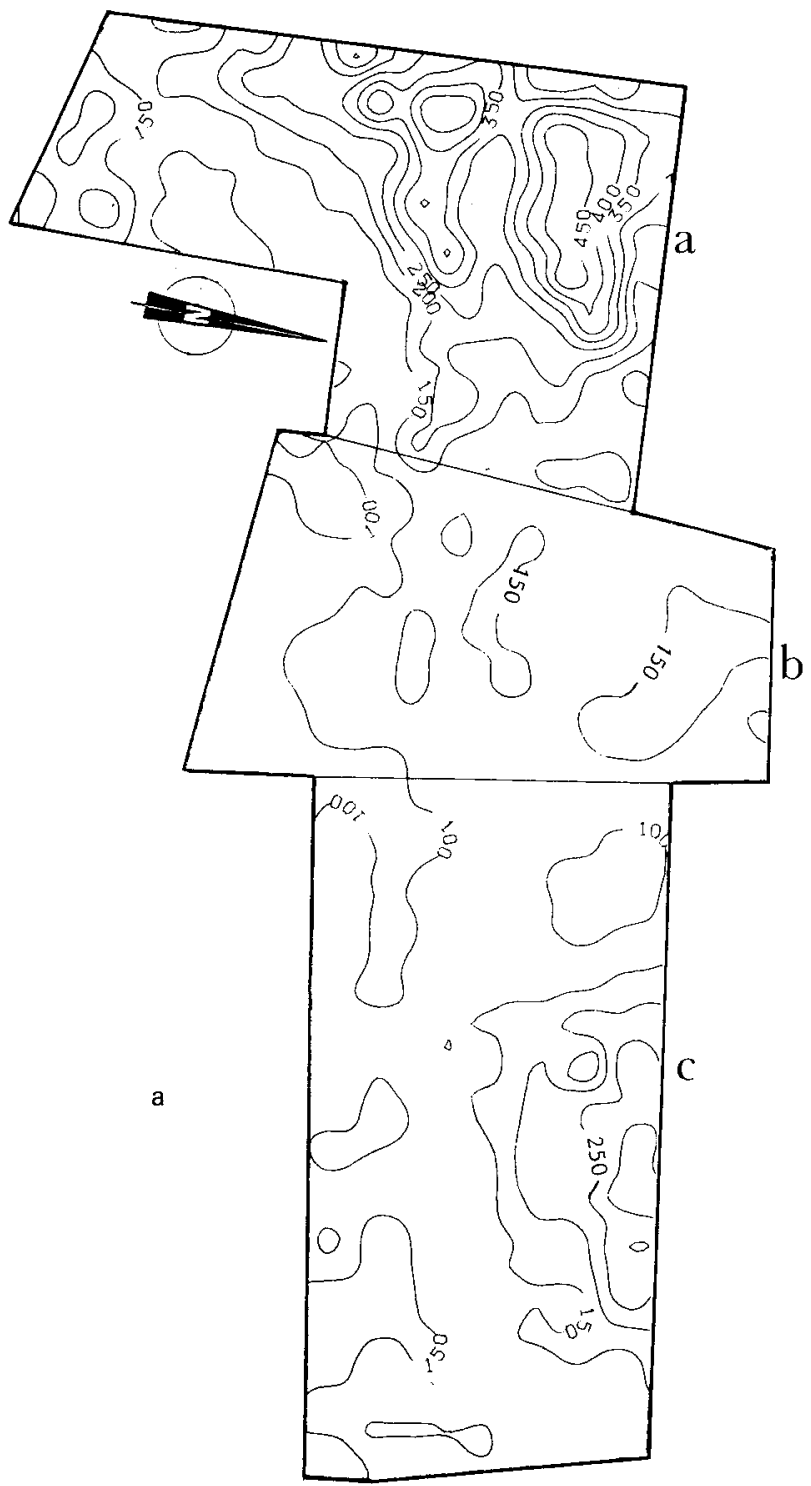

Figure 8

Carte des résistivités apparentes, mesurées à la fréquence de $711 \mathrm{kHz}$.

a) sur les données brutes (en $\Omega m$ ),

b) sur les données log normales.

$T R A, T R B$ : emplacement des transects de la figure 10.

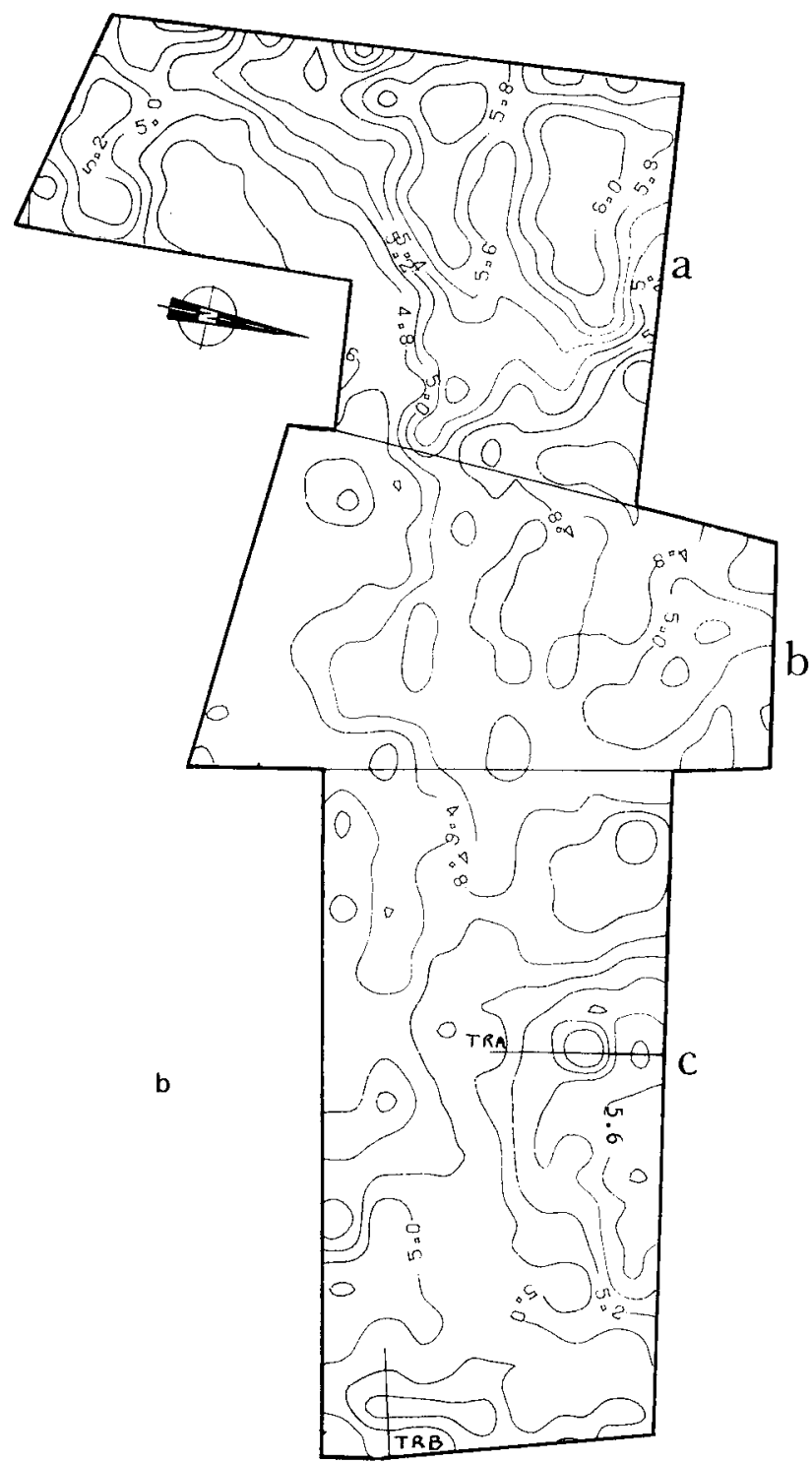

Map of apparent resistivity, measured at $711 \mathrm{kHz}$.

a) based on raw data $(\Omega m)$,

b) based on log normal data.

TRA, TRB : situation of transects of figure 10.

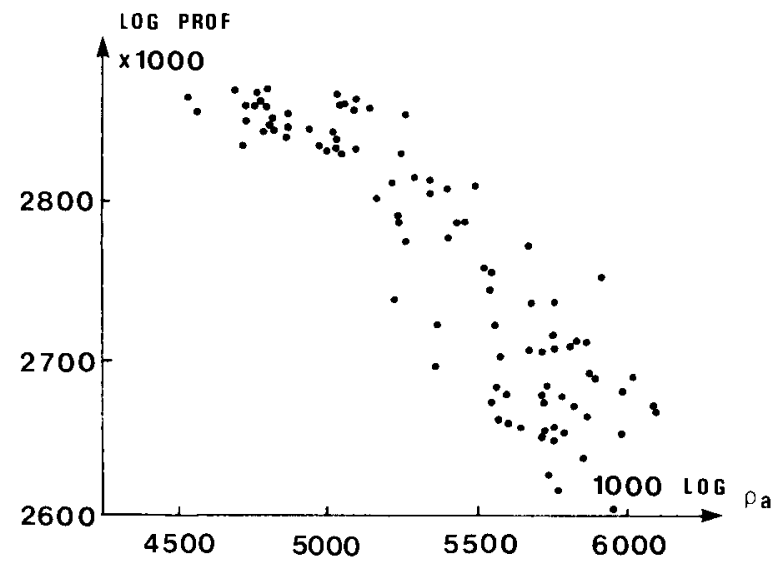

Figure 9

Corrélation entre le log de la résistivité apparente et le log de la profondeur.

Correlation between log apparent resistivity and log depth. 

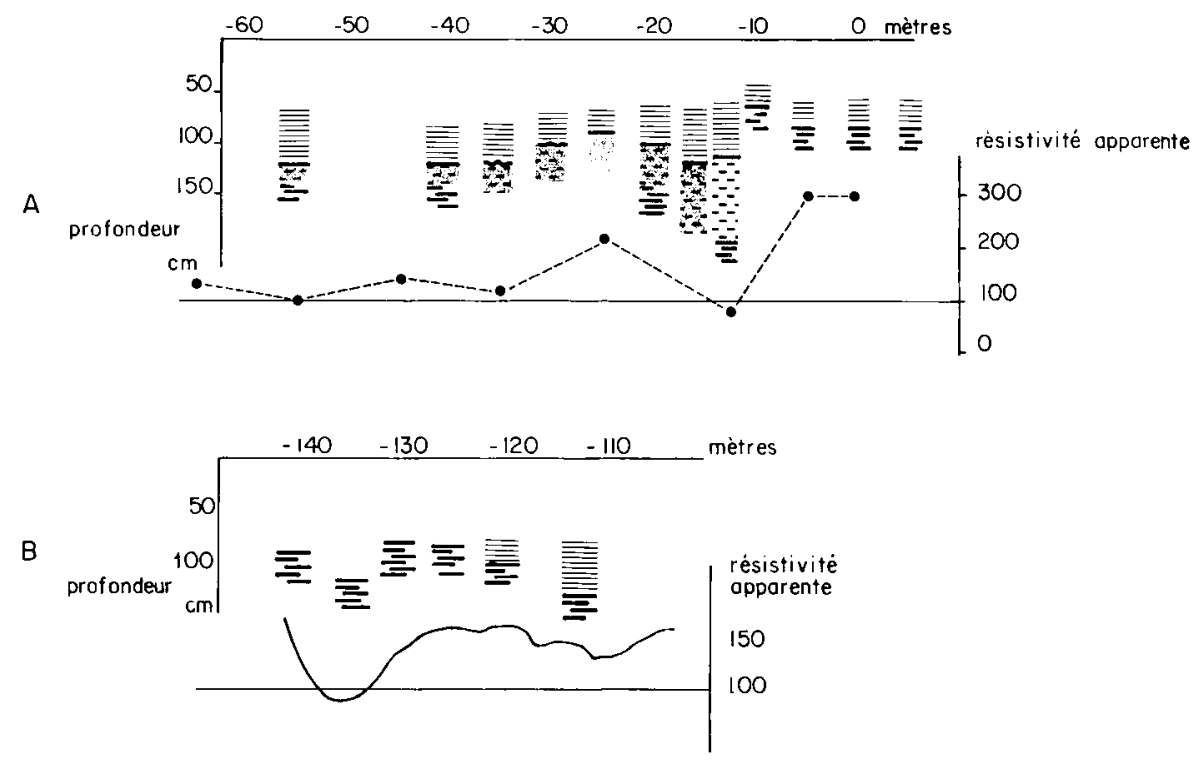

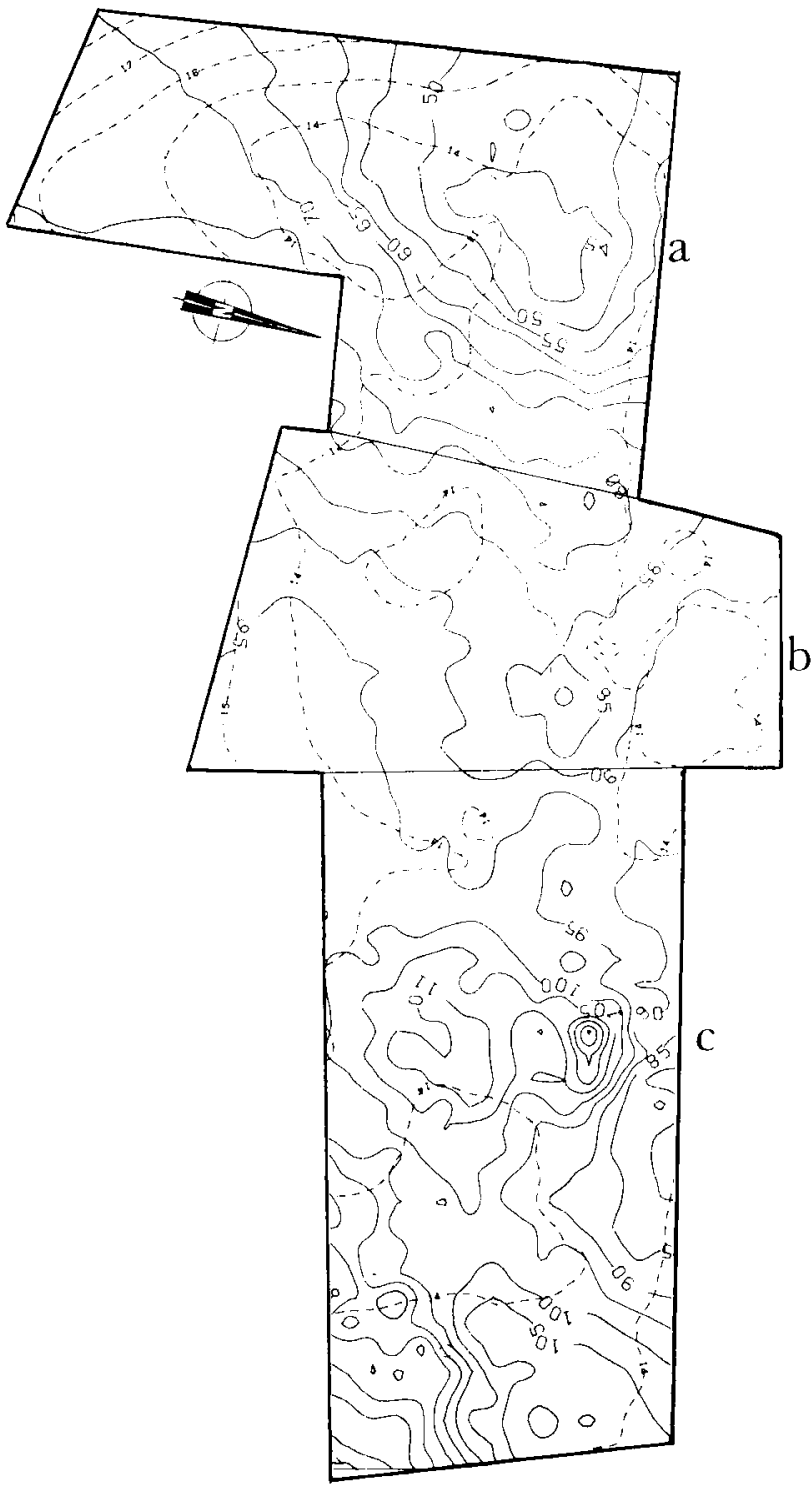

Figure 11

Carte de la profondeur du sol établie avec des données complémentaires.

Soil depth map established with complementary data.
Légende du sol :

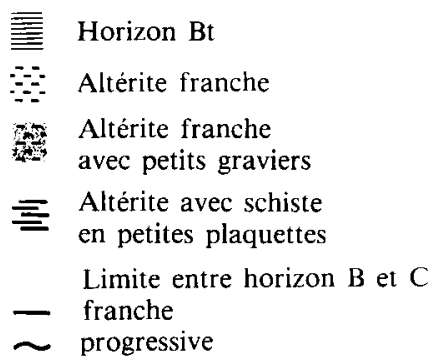

Figure 10

Comparaison sur deux transects des profils de sol et des valeurs de résistivité apparente.

Comparison of soil profiles from two transects with values of apparent resistivity.

\section{CONCLUSION}

La résistivité apparente, mesurée par M.T.A. à la fréquence de $711 \mathrm{kHz}$ sur des sols sur schiste, est reliée de façon significative à la profondeur de la discontinuité horizons superficiels/schiste ou altérite de schiste. Les conditions d'humidité du milieu, variables dans le cas étudié, ne semblent pas perturber cette réponse.

Sur une zone possédant une forte densité de sondages pédologiques la cartographie M.T.A. confirme, en affinant malgré tout les résultats, la cartographie de la profondeur du sol.

Sur une zone à faible densité de sondage pédologique, la cartographie M.T.A. remet en cause la carte de la profondeur du sol. Dans le cas étudié, elle a mis en évidence un pointement de schiste à mi-pente, pointement qui était resté inaperçu. Elle met en évidence un gradient d'approfondissement du sol partant de ce pointement dans toutes les directions. Elle a révélé des accidents extrêmement localisés du toit du schiste, de l'ordre de quelques mètres.

Cependant, elle ne permet pas de distinguer les formations superficielles, notamment la nature des horizons ou les variations au sein de l'altérite.

Cette méthode est maintenant opérationnelle pour 
l'étude des discontinuités roche saine/matériaux superficiels, même de faible profondeur, présentant un fort contraste de résistivité. Dans tous les cas où l'on a besoin d'une connaissance spatiale précise du sol, des formations superficielles et de leur contact avec la roche mère sous-jacente, cette méthode, utili- sée comme moyen d'investigation préalable, semble un outil précieux : étude de la variabilité spatiale, essais culturaux, étude sur bassin versant ou parcelle expérimentale.

Reçu le 3 juillet 1984. Accepté le 19 août 1985.

\section{ANNEXE}

\section{ANNEXE 1}

\section{Principe}

On mesure simultanément et au même endroit la composante horizontale du champ électrique $\left(\mathrm{E}_{0}\right)$ et la composante horizontale perpendiculaire du champ magnétique $\left(\mathrm{H}_{0}\right)$ pour une même fréquence de l'onde électromagnétique émise par un émetteur de radiodiffusion existant (CAGniard, 1953 ; Chevassu \& Larguillier, 1983).

- résistivité apparente :

$$
\rho_{\mathrm{a}}=\frac{1}{2 \pi \mu_{0} \mathrm{f}}\left|\frac{\mathrm{E}_{0}}{\mathrm{H}_{0}}\right|^{2}
$$

- profondeur de pénétration :

$$
p=\sqrt{\frac{\rho}{\pi \mu_{0} f}} \cong 500 \sqrt{\frac{\rho}{f}}
$$

$\rho_{\mathrm{a}}=$ résistivité en $\Omega \mathrm{m}$;

$\mathrm{f}=$ fréquence en hertz

$\mathrm{E}=$ champ électrique en $\mathrm{V} / \mathrm{m}$;

$\mathrm{H}=$ champ magnétique en $\mathrm{A} / \mathrm{m}$;

$\mu_{0}=$ perméabilité magnétique de l'air $\mu_{0}=4 \pi 10^{-7}$ henry $/ \mathrm{m}$; $\mathrm{p}=$ profondeur en $\mathrm{m}$.

\section{Problèmes liés aux courants de déplacements}

La résistivité apparente est par définition

$$
\rho_{\mathrm{a}}=\frac{1}{\mu_{0} \omega}|\mathrm{Z}|^{2}
$$

où $\mathrm{Z}=\frac{\mathrm{E}_{0}}{\mathrm{H}_{0}}$.

Application à un demi-milieu homogène de résistivité $\rho$ :

$\rho_{\mathrm{a}}=\frac{\rho}{\sqrt{1+\rho^{2} \varepsilon^{2} \varepsilon_{0}^{2} \cdot \omega^{2}}} \cdot \begin{aligned} & \text { (On ne néglige pas les courants de dépla- } \\ & \text { cement.) }\end{aligned}$

$\varepsilon=$ constante diélectrique relative ;

$\varepsilon_{0}=$ permitivité du vide $=\frac{1}{36 \pi} 10^{-9} \mathrm{farad} / \mathrm{m}$

$\omega=$ pulsation d'une onde en $\mathrm{rad} / \mathrm{s}$.

Si les courants de déplacement sont négligés, c'est-à-dire si : $\rho^{2} \varepsilon^{2} \varepsilon_{0}^{2} \omega^{2} \ll 1$.

On trouve bien : $\rho_{\mathrm{a}}=\rho$.

Sinon la formule ci-dessus permet d'apprécier l'erreur.

Prenons le cas extrême suivant :

$$
\rho=1000 \Omega \mathrm{m} ; \omega=4 \pi \cdot 10^{6} ; \mathrm{f}=2 \mathrm{MHz} ; \omega=4 .
$$

On trouve $\frac{\Delta \rho}{\rho}=0,094$
Dans le cas de l'article :

$$
\rho<200 \Omega \mathrm{m} ; \omega=2 \pi \times 711 \times 10^{3} ; \varepsilon<10 .
$$

Alors $\frac{\Delta \rho}{\rho}<0,003$.

Les courants de déplacement sont donc légitimement négligés.

3. Relation résistivité apparente/épaisseur du recouvrement et résistivité $d u$ recouvrement dans le cas d'un substratum isolant

C'est le cas du sondage M.T. 2 terrains (CAGNIARD, 1953) dans lequel $\frac{\rho_{2}}{\rho_{1}}$ est infini. Alors :

$$
\rho_{\mathrm{a}} \simeq \frac{1}{\mu_{0} \omega} \frac{\rho^{2}}{\mathrm{~h}^{2}}
$$

où $\rho$ est la résistivité du recouvrement, h son épaisseur. Cette formule n'est valable que si $\mathrm{p}>2 \mathrm{~h}$.

Si $\rho$ est constant on en tire donc :

$$
\log \rho_{\mathrm{a}}=-2 \log \mathrm{h}+\text { Constante, }
$$

d'où la nécessité de faire l'ajustement sur les log entre les 2 types de mesures.

\section{ANNEXE 2}

Théorie des variables régionalisées : aperçu sur le principe.

Une variable est dite régionalisée si elle présente à la fois un aspect aléatoire et un aspect structuré reflétant la corrélation spatiale, entre les points de mesure. L'étude de la fonction $\gamma(\mathrm{h})$, appelée variogramme, caractérise les traits structuraux de la variable étudiée :

$$
\gamma(\mathrm{h})=\frac{1}{2} \mathrm{E}\left\{[\mathrm{Z}(\mathrm{x}+\mathrm{h})-\mathrm{Z}(\mathrm{x})]^{2}\right\}
$$

où $\mathrm{E}=$ espérance mathématique ;

$\mathrm{Z} \quad$ = variable étudiée ;

$\mathrm{x} \quad=$ coordonnées du point de mesure

$\mathrm{x}+\mathrm{h}=$ coordonnées d'un point éloigné d'une distance $\mathrm{h} d \mathrm{du}$ point $\mathrm{x}$.

On recherche, à ce niveau, à ajuster au variogramme expérimental un modèle structural.

Le krigeage est une méthode d'interpolation linéaire, non biaisée, prenant comme critère d'optimalité la minimisation de la variance d'estimation. Le modèle structural choisi permettra de résoudre les équations de krigeage et de calculer les poids affectés aux données pour le calcul des estimations. 


\section{RÉFÉRENCES BIBLIOGRAPHIQUES}

Aurousseau P., 1976. - Morphologie et genèse des sols sur granite du Morvan. Thèse de Doct. Ing., Univ. de Rennes, E.N.S.A.I.N.R.A., 210 p.

Bottraud J.-C., 1983. - Résistivité électrique et étude des sols. Applications à la cartographie et à la caractérisation du fonctionnement hydrique. Thèse de Doct. Ing. U.S.T. du Languedoc, E.N.S.A. Montpellier, 191 p.

Branchard J.-P., Gascuel-Odoux C., 1981. - Recherches sur les transferts d'eau dans un petit bassin versant (Nouvoitou). Mém. D.E.A.-D.A.A., E.N.S.A. Rennes, 89 p.

Burgess T. M., Webster R., 1980. - Optimal interpolation and isorithmic mapping of soil properties. I. The semi variogram and punctual kriging. J. Soil Sci., 31 (2), 315-332.

Cagniard L., 1953. - Principe de la méthode magnéto-tellurique, nouvelle méthode de prospection géophysique. Ann. Géophys., 9, 95-125.

Chevassu G., Larguillier J.-P., 1983. - La magnéto-tellurique artificielle. Bull. Groupe fr. d'Humidimétrie neutronique, juin 1983, 13, 97-117.
Gascuel-Odoux C., 1984. - Application de la géostatistique à l'étude de la variabilité spatiale des propriétés hydriques du sol. Thèse de Doct.-Ing. de l'E.N.S.M.P. Fontainebleau, 229 p.

Gascuel-Odoux C., Merot P., Curmi P., 1982. - Approche de la variabilité spatiale de l'état et du transfert hydrique dans le sol, 87102. In: "Les colloques de l'I.N.R.A. " $n^{\circ} 15$ : Variabilité spatiale des processus de transfert dans les sols, I.N.R.A. ed., 219 p.

Guineau B., Dupis A., 1973. - Inventeurs du dispositif pour la prospection magnéto-tellurique de subsurface, Anvar Paris, brevet 7311573.

Lagabrielle R., Chevassu G., Larguillier J. F., 1983. - La magnéto-tellurique artificielle pour les projets à très faible profondeur. Bull. Assoc, Int. Géol. de l'Ingénieur, 26-27, 265-271.

Matheron G., 1965. - Les variables régionalisées et leur estimation. Masson, Paris, $305 \mathrm{p}$.

Pepin D., Garel C., 1980. Contribution à l'étude du fonctionnement hydrodynamique d'un bassin versant élémentaire sur schistes briovériens en Bretagne. Etude de la porosité d'un sol brun faiblement lessivé. Mém. D.E.A.-D.A.A., E.N.S.A.-I.N.R.A., Rennes, $69 \mathrm{p}$. 